\title{
Intramedulläre Stabilisierung und Augmentation mit unter Licht polymerisierendem Methylmethacrylat
}

Hermann Josef Bail

Stabilisierung von Frakturen mit schlechter Knochensubstanz stellt ein zunehmendes Problem in der gegenwärtigen Orthopädie und Unfallchirurgie dar. Obwohl zunehmend eine medikamentöse Prophylaxe der Osteoporose erfolgt, kommt es derzeit zu geschätzten etwa 9 Mio. osteoporotischer Frakturen weltweit [1-3]. Dazu kommt die zunehmende Anzahl von pathologischen Frakturen, größtenteils basierend auf metastasierenden malignen Grunderkrankungen [4]. Bei der Fixation von pathologischen Frakturen im Schaftbereich sind intramedulläre Implantate durch den zentralen Kraftträger und durch die minimalinvasive Applizierbarkeit vorteilhaft. Ebenso können eingeschobene winkelstabile Platten (Fixateur interne) auch bei schlechter Knochensubstanz eine suffiziente Möglichkeit zur Stabilisierung darstellen. Bei osteoporotischen Frakturen müssen die Implantate die reponierte Fraktur so lange stabilisieren, bis die Knochenheilung eingetreten ist. Das kritische Areal ist dabei das Implantat-Knochen-Interface. Bei hoher Rigidität der Implantate und geringem Widerstand seitens des Knochens kommt es in Abhängigkeit von der Belastung zur Implantatlockerung. Als zentrale Kraftträger haben intramedulläre Implantate hierbei Vorteile. Nachteilig ist dabei jedoch, dass bei zunehmend weitem Markraum im Alter der Kontakt zwischen Implantat und Knochen gering ist. Die i.d. R. wenigen und nicht winkelstabilen Verriegelungsoptionen bei intramedullären Implantaten bewirken auch hier häufig eine Überlastung des ImplantatKnochen-Interfaces [5]. Bei pathologischen Frakturen, die durch ein malignes Tumorwachstum bedingt sind, kommt es i.d.R. nicht zu einer Frakturheilung. Die Aufgabe des Implantats ist daher in vielen Fällen zur Schmerzpalliation die Stabilität der Fraktur bis zum Lebensende der Patienten zu gewährleisten. In diesen Fällen liegt i.d.R. proximal und distal der Fraktur eine insuffiziente Knochensubstanz vor, die durch Osteoporose und durch geringen Gebrauch der jeweiligen Extremität bedingt sein kann. Es existiert aufgrund dieser geschilderten Anforderungen eine Vielzahl von Publikationen, die die Verstärkung des Implantat-Knochen-Interfaces beschreiben [6-13]. Ebenso gibt es Publikationen, die die alleinige Fixation einer Fraktur durch das intramedulläre Einbringen einer aushärtenden Substanz beschreiben $[7,14,15]$. Auch wird von Versuchen berichtet, die Plattenosteosynthese durch intramedulläres Einbringen von Knochenzement zur augmentieren [16,17]. Diese Verstärkung der Fixation wird bei der sog. Verbundosteosyn- these seit langen Jahren genutzt [18-21]. Die Verbundosteosynthese wird in palliativen Situationen eingesetzt und bewirkt zusätzlich eine Kontrolle des Tumorwachstums über längere Zeit. Der Knochendefekt wird i.d.R. mit Methylmethacrylatzement aufgefüllt, häufig wird bei meta- oder diaphysären Knochendefekten der Zement auch in die angrenzenden Fragmente eingebracht und dient dort zur Verstärkung der Implantatverankerung. Die alleinige Stabilisierung mit Knochenzement ist jedoch bisher nur in Tierstudien beschrieben [15, 16, 22]. Problematisch ist bei der klinischen Anwendung bei herkömmlichem Methylmethacrylat die Kontrolle des Einbringens und der Aushärtung des intramedullären Knochenzements.

Die hier vorgestellte Implantattechnik bietet theoretisch mehrere Möglichkeiten. Zum einen kann das System ein Stand-alone-Implantat aus Methylmethacrylat produzieren, welches kontrolliert in den Markraum eingebracht und anschließend ausgehärtet wird. Durch die Anpassung an den Markraum können besonders an oberen Extremitäten wohl genügend stabile Konstrukte zur palliativen Versorgung vor allem von pathologischen Frakturen bei Metastasen erzeugt werden. Zum anderen kann das vorgestellte System bei äußerst osteoporotischen Knochen durch seine Verteilung im gesamten Markraum extramedullären Implantaten als Verstärkung dienen. Schlussendlich ist es auch möglich, das System als Ersatz für herkömmliche Verbundosteosynthesen nach Tumorresektion einzusetzen.

\section{Operationstechnik}

Das Prinzip des Implantats basiert darauf, Methylmethacrylatzement kontrolliert in den Markraum einbringen zu können und mittels Licht zur Aushärtung zu bringen. Dieses System wurde im tierexperimentellen Setting genauer vorgestellt [23] und bisher eine klinische Fallserie in einem in MEDLINE gelisteten Journal beschrieben [24]. Das sog. PBSS (Photodynamic Bone Stabilisation System), Handelsname IlluminOss (Distributor: Fa. Orthovative, Gmund am Tegernsee), wird perkutan intramedullär eingebracht. Es besteht aus einem PET-Ballon (PET: Polyethylenterephthalat, Dacron) ( $\bullet$ Abb. 1), der über eine 8-mmKnochenbohrung perkutan eingebracht wird ( $\bullet$ Abb. 2 a). Der Ballon ist mit strahlendichten Markern versehen, um 
das Einbringen kontrollieren zu können. In der neuen Version wird diese Kontrolle durch das Anbringen einer strahlendichten Spirale an den Ballon verbessert ( $\bullet$ Abb. 3). Danach wird mit einer Standardspritze ein flüssiges Monomer eingebracht (Polymethylmethacrylat, - Abb. 2b. Es erfolgt dann die exakte Reposition der Fraktur bzw. das Alignment der Knochenfragmente. Zentral im Ballon liegt ein fiberoptischer Lichtleiter. An diesen wird eine externe Lichtquelle angeschlossen, die sichtbares blaues Licht mit einer Wellenlänge von $436 \mathrm{~nm}$ appliziert ( $\triangleright$ Abb. 2 c). Abhängig von der Menge des Polymers dauert das Aushärten des Methylmethacrylats 200-800 s. An der externen Lichtquelle kann dies mit einem Timer kontrolliert werden, - Abb. 2 d). Das Zuleitungssystem wird an der Knochenoberfläche abgetrennt. Bei gutem Anpassen des Dacron-Ballons an die Form des Knochens kann theoretisch auch eine gute Rotationsstabilität erzeugt werden. Durch das perkutane Einbringen von Standardschrauben proximal und distal der Fraktur kann die Implantatstabilität erhöht werden. Ebenso kann eine hochgradige Verbesserung der Konstruktstabilität durch das Anbringen einer (winkelstabilen) submuskulär eingeschobenen Platte erzielt werden. Die Dacron-Ballons liegen in diversen Größen vor. Somit ist es möglich, eine intramedulläre Stabilisierung von Metakarpalefrakturen bis hin zu Femurfrakturen durchzuführen.

Bisher existiert nur eine geringe Zahl von Publikationen zum klinischen Einsatz des PBSS ( $\bullet$ Tab. 1). Im deutschsprachigen Raum wurden Fallserien von den Arbeitsgruppen um Pennig und Gausepohl als Abstracts publiziert. Es wurden in den beiden ersten Fallserien 10, resp. 12 Patienten mit Metakarpalefrakturen behandelt, alle Frakturen wurden zur Verheilung gebracht $[25,26]$. Zeitgleich wurde von einer biomechanischen Studie berichtet, die ebenfalls an Metakarpalia die Stabilität von winkelstabilen Platten, Osteosynthesen mit Minischrauben, intramedullären K-Drähten, externen Mini-Fixateuren, PBSS und PBSS mit Verriegelung untersucht. Die Stabilität der verschiedenen Verfahren war vergleichbar, die isolierte Osteosynthese mit dem intramedullären Methylmethacrylat war seitens der Deformation vergleichbar mit der intramedullären K-Draht-Osteosynthese [25]. In der - Tab. 1 sind bisherige Fallserien ohne Anspruch auf Voll-

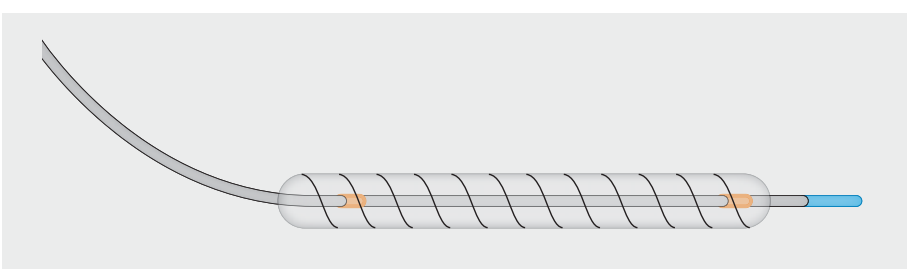

- Abb. 1 PET-Ballon mit einliegendem Lichtleiter und Kathetersystem zur Zementeinbringung.

ständigkeit aufgeführt [27-29]. Die Herstellerfirma (IlluminOss medical Inc, East Providence, Rhode Island, USA) spricht von weltweit 1500 Patienten seit 2010. Das Implantatsystem ist CE-zertifiziert, die Zulassung durch die FDA steht noch aus.

In der eigenen Anwendung kam das PBSS als supportive Maßnahme bei Plattenosteosynthese im Sinne einer Verbundosteosynthese zum Einsatz ( $\mathbf{A b b}$. $\mathbf{3}$ ).

\section{Diskussion}

Das PBSS stellt insbes. zur Augmentation von Plattenosteosynthesen und bei der Applikation an multimorbiden metastatisch erkrankten Patienten einige offensichtliche theoretische Vorteile in Aussicht. Zum einen ist die Anwendung minimalinvasiv, im palliativen Vorgehen kann somit bspw. eine eingeschobene winkelstabile Plattenosteosynthese mit dem intramedullären Methylmethacrylat augmentiert werden. Dies ist, wie in dem Fallbeispiel ( $\bullet$ Abb. 3) gezeigt, auch offen möglich. Der Vorteil liegt vor allem darin, dass im Unterschied zur Verbundosteosynthese das Methylmethacrylat als intramedulläre Schienung den gesamten Markraum des Knochens ausfüllt und im Vergleich zum eingebrachten Knochenzement eine höhere Stabilität erwarten lässt. Zum anderen kann es minimalinvasiv als Stand-alone-Implantat vor allem an den oberen Extremitäten bei pathologischen Frakturen Verwendung finden. Im Tierversuch konnte gezeigt werden, dass das Methylmethacrylat sich zumindest in der Diaphyse gut an die endostale Oberfläche des Mark-

- Tab. 1 Kennzahlen der bisher zu lluminOss publizierten Kongressbeiträge und der in MEDLINE gelisteten Publikation von Vegt et al.

\begin{tabular}{|l|l|l|l|l|}
\hline Autor & Jahr & Region & $\mathbf{n}$ & veröffentlicht in \\
\hline Gick & 2011 & Metakarpale & 10 & Abstract DGH [25] \\
\hline Heck et al. & 2011 & Metakarpale & 12 & Abstract DKOU [26] \\
\hline Heck & 2012 & Humerus & 2 & Abstract VSOU [27] \\
\hline Hoffmann et al. & 2012 & Fibula & 7 & Abstract DKOU [28] \\
\hline Gausepohl et al. & 2012 & Fibula & 13 & Poster OTA [29] \\
\hline Vegt et al. & 2014 & Radius, Ulna, Fibula, Humerus, Femur & 20 & Medical Devices: Evidence and Research [24] \\
\hline
\end{tabular}




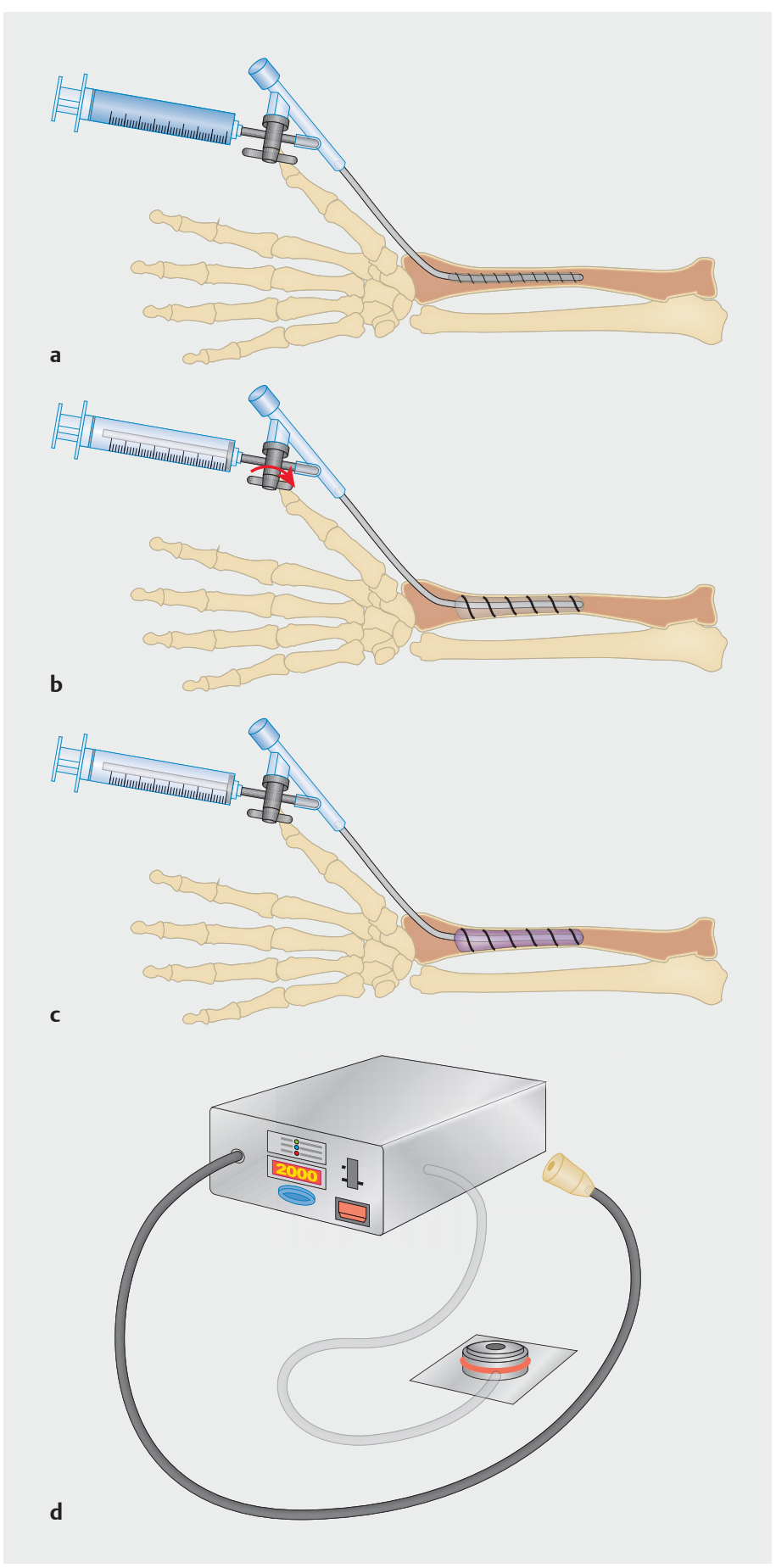

- Abb. 2 a Einbringen des zusammengefalteten Ballons mit flexiblem Lichtleiter und Befüllungskatheter am Beispiel eines distalen Radius. b Befüllen mit dem flüssigen Monomer. Der Ballon entfaltet sich, die röntgendichte Markierung wird sichtbar. c Polymerisation durch Einleiten von blauem Licht. d Lichtquelle mit Anschlusskabel und Fußschalter. raums anlegt [23]. In der gleichen Studie an Schafstibiae konnte an nicht frakturierten Schafstibiae nachgewiesen werden, dass weder der trabekuläre Knochen in der Metaphyse noch die endostale Oberfläche geschädigt werden. Hier bietet das System den Vorteil, dass es im Vergleich zum herkömmlichen Methylmethacrylat bei deutlich geringeren Temperaturen aushärtet (PMMA $100^{\circ} \mathrm{C}$; PBSS62 ${ }^{\circ} \mathrm{C}$ ). Auch ist die Aushärtungszeit im Vergleich zum herkömmlichen Knochenzement (PMMA, 27 min) beim PBSS deutlich kürzer (4 min) [24]. Die Aushärtung unter blauem Licht findet auch in der Knochenhistologie Verwendung, sie hat dort den Vorteil, dass unentkalkte Hartschnitte angefertigt werden können und durch die geringe Hitzeentwicklung Proteinstrukturen für weitere histologische Verfahren (z. B. Immunhistologie) erhalten bleiben. Ebenso konnte die genannte Tierstudie zeigen, dass trotz des ausgefüllten Markraums eine Osteotomie ungestört verheilen kann, der radiolisch und histologisch kontrollierte Ablauf der Verheilung ist vergleichbar mit demjenigen einer Osteotomie ohne PBSS im Markraum. So konnte z. B. keine Hemmung der endostalen Knochenformation und Vaskularisierung mit den PBSS-Implantaten gesehen werden. Hinsichtlich der Biokompatibilität gibt es beim Tierexperiment keine Hinweise darauf, dass die Frakturheilung gestört wird. Ein klarer Nachteil des Systems, vor allem bei jungen Patienten, besteht jedoch in der fehlenden Resorbierbarkeit. Auch stellt die unklare Explantierbarkeit möglichweise ein Problem bei der Behandlung junger Patienten dar. Es ist zu vermuten, dass bei Aufweitung des Ballons metaphysär eine problemlose Entfernung des PBSS-Polymerstabs über den Isthmus nicht möglich ist.

Es gibt sowohl experimentelle als auch klinische Studien zur Augmentation von Implantaten und Frakturen mit Kalziumphosphatzementen [17,30,31]. Die Biokompartibilität und Resorption mit sogar vorhandener osteogener Potenz von Kalziumphosphatzementen konnte in entsprechenden Studien gezeigt werden [32,33]. Allerdings sind Kalziumphosphatzemente durch ihre biomechanischen Eigenschaften bisher nicht in der Lage, als intramedulläre Kraftträger zu fungieren, vielmehr wurden diese bisher als supportive Materialien vor allem bei Gelenkfrakturen eingesetzt.

In der größten bisher publizierten Fallserie zur Nutzung des PBSS [24] wurden insgesamt 41 Frakturen versorgt. In diesen Anwendungsbeobachtungen wurden viele verschiedene Frakturarten (osteoporotische Frakturen, Frakturen bedingt durch Metastasen, drohende Frakturen aufgrund von Metastasen) und viele verschiedene anatomische Regionen (distaler Radius, Ulna, distale Fibula, Humerus und Femur) behandelt. In den meisten Fällen wurde PBSS als singuläres Implantat verwendet. Bei 6 Patienten erfolgte die Versorgung mit PBSS und zusätzlichen Schrauben an der Fibula, desgleichen bei 4 Patienten am Humerus. Bei 2 Patienten (einmal distale Fibula, 
einmal Humerus) erfolgte die Versorgung mit PBSS und einer Platte. Im Fall von akuten Frakturen kam es in allen Fällen zu einem regelhaften Heilungsverlauf. Ein Vorteil des PBSS könnte auch sein, dass das Elastizitätsmodul des Polymers (1,5 GPa) vergleichbar mit dem von trabekulärem Knochen ist (0,1-4,5 GPa) [24]. Im Gegensatz dazu haben Titan (115 GPa) und Stahl (195 GPa) ein ungleich höheres Elastizitätsmodul, welches zur Überlastung des Knochens am Implantat-Knochen-Interface führen kann. In allen in der $>$ Tab. 1 beschriebenen Fallserien wurden die Patienten, an denen Frakturen oder Osteolysen der oberen Extremitäten behandelt wurden, ohne Cast oder Orthese behandelt. Dies ist insbes. bei multimorbiden Patienten ein großer Vorteil. Bei Frakturen der unteren Extremitäten wurde i.d. R. für 2 Wochen ein Softcast angelegt mit der Freigabe zur Belastung ab 2 Tage postoperativ.

Das System bietet somit folgende potenzielle Vorteile gegenüber anderen Verfahren:

- minimalinvasiv

- definierte Monomerverteilung durch den Dacron-BalIon

- Aushärtungszeitpunkt durch den Chirurgen frei wählbar durch Lichtapplikation (nach guter Reposition)

- Augmentierung des gesamten Markraums bei Nutzung als supportives Verfahren

- zusätzliche Stabilität durch frei wählbare perkutane Verriegelung

- mit spongiösem Knochen vergleichbares Elastizitätsmodul

- geringere Temperaturentwicklung im Vergleich zum herkömmlichen Knochenzement

- keine Verteilung des Zements in die periostale Heilungszone

Zu berücksichtigen sind als möglicherweise nachteilig:

- fehlende Resorbierbarkeit insbes. bei der Anwendung an jungen Patienten

- unklare Explantatierbarkeit des Implantats

\section{Zusammenfassung}

Bei der Anwendung des PBSS kann unter kontrollierten Bedingungen minimalinvasiv eine intramedulläre Schienung mit einem Methylmethacrylatpolymer erfolgen. Diese zeigt vielversprechende theoretische Vorteile auf. Die fehlende Resorbierbarkeit und die zu erwartenden Probleme bei der Entfernung sprechen gegen den Einsatz bei „normalen“ Frakturen bei jüngeren Patienten. Bei pathologischen Frakturen aufgrund von Osteoporose und bei metastatisch bedingten Frakturen können sie an oberen Extremitäten sowohl als Stand-alone-Implantate als auch in Kombination mit anderen Implantaten (Schrauben zur Verriegelung, Platten, Fixateur interne) eine vielversprechende Möglichkeit zur Verbesserung

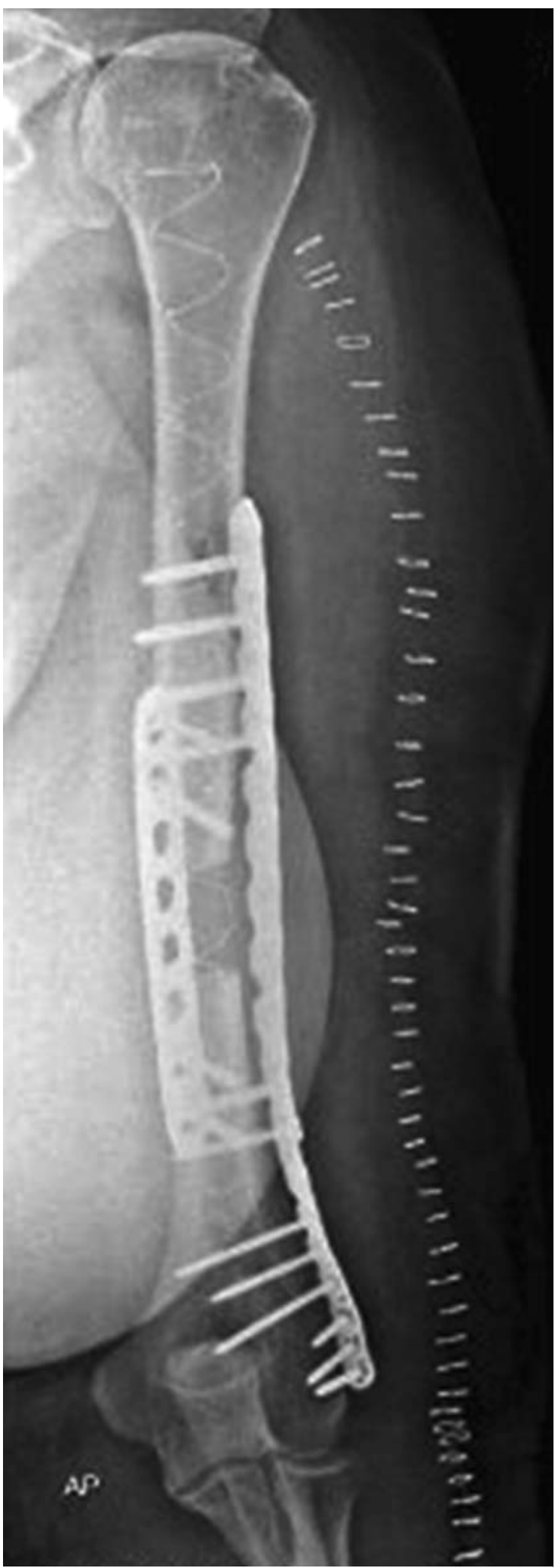

- Abb. 3 Doppelplattenosteosynthese nach Resektion einer Metastase eines Mammakarzinoms aus dem Humerusschaft. Verbundosteosynthese mit IlluminOss-System intramedullär (erkennbar an der röntgendichten spiralförmigen Markierung im Markraum). 
der Frakturfixation und Mobilisierung der Patienten darstellen. An den unteren Extremitäten bieten sie eine Möglichkeit zur Augmentation vor allem bei eingeschobenen Plattenosteosynthesen (Fixateur interne). Den bisherigen Publikationen mangelt es an Vergleichbarkeit, die geringe Komplikationsrate und die guten Ergebnisse müssen mit Zurückhaltung beurteilt werden. Es ist zu erwarten, dass bei Zulassung durch die FDA vor allem in den USA eine starke Zunahme an Applikationen folgen wird. Es ist zu hoffen, dass das System in adäquaten Studien höherer Evidenz seine zu erwartenden Vorteile und Beweis stellen kann.

\section{Interessenkonflikt}

Der Autor gibt an, dass kein Interessenkonflikt besteht.

Über die Autoren

\section{Hermann Josef Bail}

Univ.-Prof. Dr. med., Leitender Arzt, Universitätsklinik für Orthopädie und Unfallchirurgie, Klinikum Nürnberg - Paracelsus Medizinische Privatuniversität

Korrespondenzadresse

Univ.-Prof. Dr. med. Hermann Josef Bail

Leitender Arzt

Universitätsklinik für Orthopädie und Unfallchirurgie

Klinikum Nürnberg - Paracelsus Medizinische Privatuniversität Breslauer Straße 201

90471 Nürnberg

Hermann-Josef.Bail@klinikum-nuernberg.de

\section{Literatur}

[1] Costa AG, Wyman A, Siris ES et al. When, where and how osteoporosis-associated fractures occur: an analysis from the Global Longitudinal Study of Osteoporosis in Women (GLOW). PLoS One 2013; 8: e83306

[2] Johnell O, Kanis JA. An estimate of the worldwide prevalence and disability associated with osteoporotic fractures. Osteoporos Int 2006; 17: 1726-1733

[3] Hernlund E, Svedbom A, Ivergård M et al. Osteoporosis in the European Union: medical management, epidemiology and economic burden. A report prepared in collaboration with the International Osteoporosis Foundation (IOF) and the European Federation of Pharmaceutical Industry Associations (EFPIA). Arch Osteoporos 2013 8: 136

[4] Ratasvuori M, Wedin R, Keller J et al. Insight opinion to surgically treated metastatic bone disease: Scandinavian Sarcoma Group Skeletal Metastasis Registry report of 1195 operated skeletal metastasis. Surg Oncol 2013; 22: 132-138

[5] Changulani M, Jain UK, Keswani T. Comparison of the use of the humerus intramedullary nail and dynamic compression plate for the management of diaphyseal fractures of the humerus. A randomised controlled study. Int Orthop 2007; 31: 391-395
[6] Kammerlander C, Neuerburg C, Verlaan J] et al. The use of augmentation techniques in osteoporotic fracture fixation. Injury 2016; 47 (Suppl. 2): S36-S43

[7] Larsson S, Bauer TW. Use of injectable calcium phosphate cement for fracture fixation: a review. Clin Orthop Relat Res 2002 395: 23-32

[8] Larsson S, Procter P. Optimising implant anchorage (augmentation) during fixation of osteoporotic fractures: is there a role for bone-graft substitutes? Injury 2011; 42 (Suppl. 2): S72S76

[9] Mattsson P, Larsson S. Stability of internally fixed femoral neck fractures augmented with resorbable cement. A prospective randomized study using radiostereometry. Scand I Surg 2003; 92: 215-219

[10] Wähnert D, Lange JH, Schulze M et al. The potential of implant augmentation in the treatment of osteoporotic distal femur fractures: a biomechanical study. Injury 2013; 44: 808-812

[11] Goetzen M, Nicolino T, Hofmann-Fliri L et al. Metaphyseal screw augmentation of the LISS-PLT plate with polymethylmethacrylate improves angular stability in osteoporotic proximal third tibial fractures: a biomechanical study in human cadaveric tibiae. J Orthop Trauma 2014; 28: 294-299

[12] Wähnert $D$, Lange JH, Schulze $M$ et al. A laboratory investigation to assess the influence of cement augmentation of screw and plate fixation in a simulation of distal femoral fracture of osteoporotic and non-osteoporotic bone. Bone Joint J 2013; 95-B: $1406-1409$

[13] Ross RD, Hamilton JL, Wilson BM et al. Pharmacologic augmentation of implant fixation in osteopenic bone. Curr Osteoporos Rep 2014; 12: 55-64

[14] Lind PJ, Degernes LA, Olson DE et al. Bone cement/polypylene rod orthopedic technique. J Assoc Avian Vet 1989; 3: 203-205

[15] Putney SD, Borman E, Lohse C. Methylmethacrylate fixation of avian humeral fractures. J Am Anim Hosp Assoc 1983; 19: 773-782

[16] Kuzma AB, Hunter B. A new technique for avian fracture repair using intramedullary polymethylmethacrylate and bone plate fixation. I Am Anim Hosp Assoc 1991; 27: 239-248

[17] Lobenhoffer P, Gerich T, Witte F et al. Use of an injectable calcium phosphate bone cement in the treatment of tibial plateau fractures: a prospective study of twenty-six cases with twenty-month mean follow-up. J Orthop Trauma 2002; 16: 143-149

[18] Schwabe P, Ruppert M, Tsitsilonis S et al. Surgical management and outcome of skeletal metastatic disease of the humerus. Acta Chir Orthop Traumatol Cech 2014; 81: 365-370

[19] Holz U. [Compound osteosynthesis in spontaneous femoral fractures]. Aktuelle Traumatol 1985; 15: 100-103

[20] Friedl W, Ruf W, Mischkowsky T. [Compound double plate osteosynthesis in subtrochanteric pathologic fractures. A clinical and experimental study]. Chirurg 1986; 57: 713-718

[21] Wessling M, Pflugmacher R, Müller MC et al. [Basic principles of surgical treatment of bone metastases]. Z Orthop Unfall 2013; 151: 303-314

[22] Mirzasadeghi A, Narayanan SS, $\mathrm{Ng} \mathrm{MH}$ et al. Intramedullary cement osteosynthesis (IMCO): a pilot study in sheep. Biomed Mater Eng 2014; 24: 2177-2186

[23] Zani BG, Baird R, Stanley JR et al. Evaluation of an intramedullary bone stabilization system using a light-curable monomer in sheep. J Biomed Mater Res B Appl Biomater 2016; 104: 291 299 
[24] Vegt P, Muir JM, Block JE. The Photodynamic Bone Stabilization System: a minimally invasive, percutaneous intramedullary polymeric osteosynthesis for simple and complex long bone fractures. Med Devices (Auckl) 2014; 12: 453-461

[25] Gick S. Intramedullary Polymer Osteosynthesis (IlluminOss): Biomechanical comparative tests and initial clinical experience with metacarpal bone fractures. DGH-Congress (German Association for Surgery of the Hand) - Bonn, October 7, 2011

[26] Heck S, Gick S, Rabiner R et al. Innovative intramedullary stabilization of metacarpal shaft fractures - initial clinical experiences with a photodynamic polymer (IlluminOss). DKOU 2011 (German Congress for Orthopedics and Accident Surgery) - Berlin, October 26, 2011

[27] Heck S. Treatment of fractures caused by osteoporosis. VSOU Baden-Baden (Association of South German Orthopedists and Trauma Surgeons) Annual Meeting, April 2012

[28] Hoffmann F, Gausepohl T. Minimally invasive stabilization of weber b fractures using intramedullary balloon stabilization (IlluminOss) in elderly patients. DKOU - German Congress of Orthopedics and Trauma Surgery, October 25, 2012
[29] Gausepohl T, Rashid A, Baluch M et al. Ankle fracture fixation with a novel intramedullary photodynamic polymeric rod: a case series review. Orthopaedic Trauma Association 28th Annual Meeting, October 3-6, 2012

[30] Cohen MS, Whitman K. Calcium phosphate bone cement - the Norian skeletal repair system in orthopedic surgery. AORN J 1997; 65: 958-962

[31] Simpson D, Keating JF. Outcome of tibial plateau fractures managed with calcium phosphate cement. Injury 2004; 35 : 913-918

[32] Theiss F, Apelt D, Brand B et al. Biocompatibility and resorption of a brushite calcium phosphate cement. Biomaterials 2005; 26: 4383-4394

[33] Link DP, van den Dolder J, van den Beucken J] et al. Evaluation of the biocompatibility of calcium phosphate cement/PLGA microparticle composites. J Biomed Mater Res A 2008; 87: 760-769

Bibliografie

DOI http://dx.doi.org/10.1055/s-0043-101723

OP-JOURNAL 2017; 33: 72-77 (c) Georg Thieme Verlag KG Stuttgart · New York ISSN 0178-1715 